\title{
Research on Loss in Power Grid Induced by Three-Phase Imbalance
}

\author{
Yunxia Dong \\ School of Electrical and Electronic Engineering, North China Electrical Power University, Beijing, China
}

\section{Email address:}

dyx2007@126.com

\section{To cite this article:}

Yunxia Dong. Research on Loss in Power Grid Induced by Three-Phase Imbalance. Journal of Electrical and Electronic Engineering. Vol. 8, No. 3, 2020, pp. 103-108. doi: 10.11648/j.jeee.20200803.14

Received: June 19, 2020; Accepted: July 31, 2020; Published: August 10, 2020

\begin{abstract}
The power quality problems in the power grid have some direct impact on the distribution network such as to increase the system's active power loss. There are many single-phase power users in the power distribution network which will inevitably cause the power quality problems such as three-phase imbalance. Three phase imbalance is widespread across the power grid and causes additional loss in the transformer and the transmission line. Therefore, the loss of the low-voltage distribution network is also increased rapidly, so it is of great significance to consider the loss of low-voltage distribution network under the disturbance of such a power quality problem. In this paper, the calculation method of the additional losses of the transmission line and the transformer in three-phase imbalance is derived theoretically. The accuracy of theoretical formula is verified in MATLAB/SIMULINK simulation platform. Secondly, the simulation data of the additional loss generated in the transformer and the transmission line when the three-phase imbalance exists is comprehensively analyzed. Several cases of different unbalanced loads are considered in the paper. The additional value of the power loss relative to the balanced case which is caused by the three phase imbalance is the largest one for the case in which phase A and B have heavy load and phase C is light load and the absolute value of the power loss in this case is also the largest in three cases. The additional loss is not proportional to the total loss both for the transformer and the line. We verify that the additional loss caused by three-phase imbalance is dependent on the unbalanced degree. As the unbalanced degree is increasing, both the absolute value of the additional loss and the increment ratio of loss for the transformer and the line are enhanced simultaneously.
\end{abstract}

Keywords: Three-Phase Imbalance, Transmission Line, Transformer, MATLAB/SIMULINK Simulation, Additional Loss

\section{Introduction}

As various nonlinear and impact loads are widely used in modern society, more and more power quality problems have emerged in the power grid. The most direct impact of the power quality problem on the distribution network is to increase the system's active power loss. The power loss of low-voltage distribution network accounts for almost one half of the total loss of the power distribution network. The increase of the additional loss in the low-voltage distribution network caused by the power quality problems directly affects the operating efficiency of the power grid companies [1-3].

At present, more research focuses on the analysis of the impact of harmonics and three-phase imbalance on the low voltage distribution network and the corresponding control measures. Literature [4] analyzes the effect of harmonics on the losses of low-voltage distribution networks. It uses
Norton's equivalent method to model and analyze the nonlinear loads of distribution networks. Literature [5] describes the effects of three-phase imbalance on the losses of low-voltage distribution networks. A new method of network loss calculation is proposed, which uses the sequence components of three-phase unbalanced current to solve the total loss of the distribution network. The literature [6] analyzes the effect of the transformer three-phase load asymmetry on the line loss ratio of the low-voltage distribution network. The study on the power quality issues of the low-voltage distribution network loss is not very thorough and most of the existing studies have studied the loss of the low-voltage distribution network when a certain kind of power quality exists [7-13]. However, the additional loss of the actual low-voltage distribution network which is caused by the three-phase imbalance is deserved to study carefully. Therefore, it is of great significance to study the relation 
between the additional losses of low-voltage distribution network and the three-phase imbalance degree.

The remainder of this paper is organized as follows: we first deduce the additional losses of the transmission line and the transformer when the three-phase imbalances exist and then study deeply the additional losses in the low-voltage distribution network in Section 2. To verify the validity and rationality of the theoretical derivation, we consider several cases which are simulated and verified in the MATLAB software, and the effect of three-phase imbalance on the additional loss of key equipment is analyzed in Section 3. Furthermore, the correlation between the additional loss of the transmission line and the transformer and the three-phase unbalanced degree is studied. We give the conclusion of the paper in Section 4.

\section{Calculation of Additional Power Loss Under Three Phase Imbalance}

In the electric power system, the amplitude and phase angle of the three-phase voltage and current are asymmetrical due to three-phase unbalanced load. The phenomenon is called three phase imbalance.

According to the national standard, the three-phase unbalanced degree is expressed as the square root mean value of the positive sequence fundamental wave component and the negative sequence fundamental wave component or the zero-sequence fundamental wave component of the current. But this representation method is not intuitive enough to calculate the loss of the devices. In this paper, we express the unbalanced degree of each phase referring to IEEE std112-1991 to analyze the influence of the three-phase unbalance on the loss of the low voltage distribution network, so it can be more intuitively characterized the internal relations between the additional loss of the equipment and the three-phase unbalanced degree [7].

The following formula is the definition of the three-phase current unbalanced degree:

$$
\beta_{\varphi}=\frac{I_{\varphi}-I_{\mathrm{av}}}{I_{a v}} \times 100 \%
$$

At the same time, the maximal unbalanced coefficient is introduced as follows:

$$
\beta_{\max }=\frac{\max \left\{\left|I_{A}-I_{a v}\right|,\left|I_{B}-I_{a v}\right|,\left|I_{C}-I_{a v}\right|\right\}}{I_{a v}}
$$

In the upper formulas: $I_{\varphi}$ is the current root mean square (RMS) of each phase ( $\varphi=A, B, C) ; I_{\mathrm{av}}$ is the mean value of the effective value of the three-phase current, which is: $I_{a v}=\frac{I_{A}+I_{B}+I_{C}}{3}$.

When the amplitude and phase angle of three-phase current are unbalanced, the currents of the three phases can be expressed as:

$$
\left\{\begin{array}{l}
\dot{I}_{A}=I_{a v}\left(1+\beta_{A}\right) \angle \phi_{A} \\
\dot{I}_{B}=I_{a v}\left(1+\beta_{B}\right) \angle \phi_{B} \\
\dot{I}_{C}=I_{a v}\left(1+\beta_{C}\right) \angle \phi_{C}
\end{array}\right.
$$

\subsection{Additional Loss of Distribution Transformer}

There are many single-phase and two-phase loads in the low voltage distribution network and the asymmetric three-phase load result in unbalanced currents in the system. In three-phase unbalanced operation condition, the transformer and transmission line generate more active power loss, transformer output capacity reduction, power supply quality reduction and other adverse consequences. Three-phase imbalance should be considered when the amplitude and phase angle do not meet the three-phase equilibrium condition, and the additional loss of distribution transformer and line will be calculated [8].

When the three-phase winding has balanced current, the transformer winding loss can be expressed as:

$$
P_{T 1}=3 \times\left[\left(I_{A}+I_{B}+I_{C}\right) / 3\right]^{2} \times R_{T}=3 I_{a v 0}^{2} R_{T}
$$

When the three-phase winding current is unbalanced, the transformer winding loss can be expressed as:

$$
\begin{aligned}
P_{T 2} & =\left(I_{A}^{2}+I_{B}^{2}+I_{C}^{2}\right) \times R_{T} \\
& =I_{a v}^{2}\left[\left(1+\beta_{A}\right)^{2}+\left(1+\beta_{B}\right)^{2}+\left(1+\beta_{C}\right)^{2}\right] R_{T}
\end{aligned}
$$

In the calculation of three-phase unbalanced loss of low voltage distribution network, the additional iron loss of transformer is usually ignored. Owing to $\beta_{A}+\beta_{B}+\beta_{C}=0$, the additional loss of low voltage distribution transformer can be expressed as:

$$
\begin{aligned}
\Delta P_{T} & =P_{T 2}-P_{T 1} \\
& =I_{a v}^{2}\left[\beta_{A}^{2}+\beta_{B}^{2}+\beta_{C}^{2}+3\right] R_{T}-3 I_{a v 0}^{2} R_{T} \\
& =K_{T} I_{a v}^{2} R_{T}-3 I_{a v 0}^{2} R_{T}
\end{aligned}
$$

Where: $K_{\mathrm{T}}$ represents the three phase unbalanced coefficient of the transformer.

Equation (6) is the additional loss model of distribution transformer under unbalanced three-phase current.

\subsection{Additional loss of Transmission Line}

When the three-phase load is balanced, the current flowing through the neutral line is zero, and there is no power loss on the neutral line. Therefore, it is not necessary to calculate the loss on the neutral line. When the three-phase current is unbalanced, the line will not only cause additional losses on each phase line, but the loss on the neutral line should be also 
considered [9].

First we consider the loss on the phase line of low voltage distribution network. The loss on three phases which is caused by three-phase imbalance can be expressed as:

$$
\Delta P_{L}=I_{a v}^{2}\left(\beta_{A}^{2}+\beta_{B}^{2}+\beta_{C}^{2}+3\right) R_{L}-3 I_{a v 0}^{2} R_{L}=K_{L}-3 I_{a v 0}^{2} R_{L}
$$

Now we calculate the neutral line loss of low voltage distribution network. When the three-phase load is unbalanced, the current of the neutral line of the three-phase four-wire low-voltage distribution network is:

$$
\begin{aligned}
\dot{I}_{0} & =\dot{I}_{A}+\dot{I}_{B}+\dot{I}_{C} \\
& =I_{a v}\left[\left(1+\beta_{A}\right) \cos \phi_{A}+\left(1+\beta_{B}\right) \cos \phi_{B}+\left(1+\beta_{C}\right) \cos \phi_{C}\right] \\
& +j I_{a v}\left[\left(1+\beta_{A}\right) \sin \phi_{A}+\left(1+\beta_{B}\right) \sin \phi_{B}+\left(1+\beta_{C}\right) \sin \phi_{C}\right]
\end{aligned}
$$

Make $a=\left(1+\beta_{A}\right) \cos \phi_{A}+\left(1+\beta_{B}\right) \cos \phi_{B}+\left(1+\beta_{C}\right) \cos \phi_{C}$ and $b=\left(1+\beta_{A}\right) \sin \phi_{A}+\left(1+\beta_{B}\right) \sin \phi_{B}+\left(1+\beta_{C}\right) \sin \phi_{C}$. The loss of the neutral line can be expressed as:

$$
\Delta P_{0}=I_{0}^{2} R_{0}=\left(a^{2}+b^{2}\right) I_{a v^{R} 0}^{2}
$$

The additional loss of the three-phase four-wire distribution line is:

$$
\begin{aligned}
\Delta P_{\text {Lall }} & =\left(\beta_{A}^{2}+\beta_{B}^{2}+\beta_{C}^{2}+3\right) I_{a v}^{2} R_{L}-3 I_{a v 0}^{2} R_{L}+\left(a^{2}+b^{2}\right) I_{a v}^{2} R_{0} \\
& =K_{L} I_{a v}^{2} R_{L}-3 I_{a v 0}^{2} R_{L}+K_{0} I_{a v}^{2} R_{0}
\end{aligned}
$$

Among them, $K_{\mathrm{L}}$ represents the three-phase unbalance coefficient of three phase line, and the $K_{0}$ represents the three-phase unbalance coefficient of the neutral line.

The above Equation shows that the additional loss of the distribution line is greater when the three-phase unbalance coefficient and the three-phase unbalance coefficient of the neutral line become larger, and the additional loss of the distribution line is proportional to the two coefficients $K_{\mathrm{L}}$ and $K_{0}$.

To obtain the quantitative relationship between the additional loss of low-voltage distribution network and the indicators three-phase unbalanced degree, the concept of loss increment ratio of the transformer is given in this paper:

$$
\delta_{T}=\frac{\Delta P_{T}}{P_{T}} \times 100 \%
$$

Where: $\Delta \mathrm{P}_{\mathrm{T}}$ is the additional loss of the transformer when the system is unbalanced; $\mathrm{P}_{\mathrm{T}}$ is the total loss of the transformer.

The loss increment ratio of the transmission line is

$$
\delta_{L}=\frac{\Delta P_{L}}{P_{L}} \times 100 \%
$$

Where: $\Delta \mathrm{P}_{\mathrm{L}}$ is the additional loss of the line when the system is unbalanced; $\mathrm{P}_{\mathrm{L}}$ is the total loss of the line.

\section{Theoretical Result and Simulation Analysis of Additional Losses}

It can be seen from the above formula that the additional loss of the low-voltage distribution network under the condition of the power quality disturbance is closely related to the unbalanced degree of the currents. In the simulation software MATLAB, a simulation system is set up with the simplified model to verify the correctness of the proposed formula. Then, the overall power loss of the system is calculated for several situations with the different unbalanced degree of the load.

In low-voltage distribution network, the transformers are often used to reduce the $10 \mathrm{kV}$ voltage level to $0.4 \mathrm{kV}$ voltage level and supply power to customers through three-phase four-wire distribution, so this simulation also uses $0.4 \mathrm{kV}$ distribution system. Because the circuit of the low-voltage distribution network is very complex, the equivalent resistance method is used to configure the main equipment in the distribution network. The simulation system sets the same three-phase impedance, and the neutral line resistance is equal to the line resistance. Under the condition of constant total load, the three-phase load distribution ratio is adjusted step by step, and the loss under different unbalance degree is obtained.

The equivalent distribution network is shown in the diagram [13]. The equivalent impedance of the transformer is converted to the low voltage side in the diagram, where the equivalent impedance of the line is taken at the same time. We can directly analyze the relationship between power quality problems and equipment additional losses. The three-phase impedance of the setting system is the same, and the neutral line resistance is assumed to be equal to the resistance of the line. Under the condition that the total load is constant, the distribution ratio of load between three phases is gradually adjusted, and the valued of the power loss under different unbalanced degrees is obtained $[14,15]$

Figure 1 shows the equivalent resistance model for the low-voltage distribution network. According to the different situations for the different unbalanced loads, the three-phase imbalance of the low-voltage distribution network can be roughly divided into three situations. The three cases are respectively: one-phase is heavy-duty, two-phases are light loads (case I), one-phase is light load, two-phases are heavy loads (case II), and one-phase load is averaging other two phases which are one heavy load phase and one light load phase (case III). As the three-phase imbalance mainly affects the copper loss of the low-voltage distribution network, only the additional copper consumption is analyzed here. Table 1 shows the three-phase load conditions of load A, B and C. There are three cases which are analyzed here. Three-phase equilibrium case is set for all the three phases load is $20 \mathrm{kVA}$. The additional loss equals the loss in the unbalanced case minus the loss in the balanced case. The additional loss means the loss in the unbalanced case minus the loss of the equilibrium case. Then the additional loss represents the effect of the unbalanced degree to the power loss. 


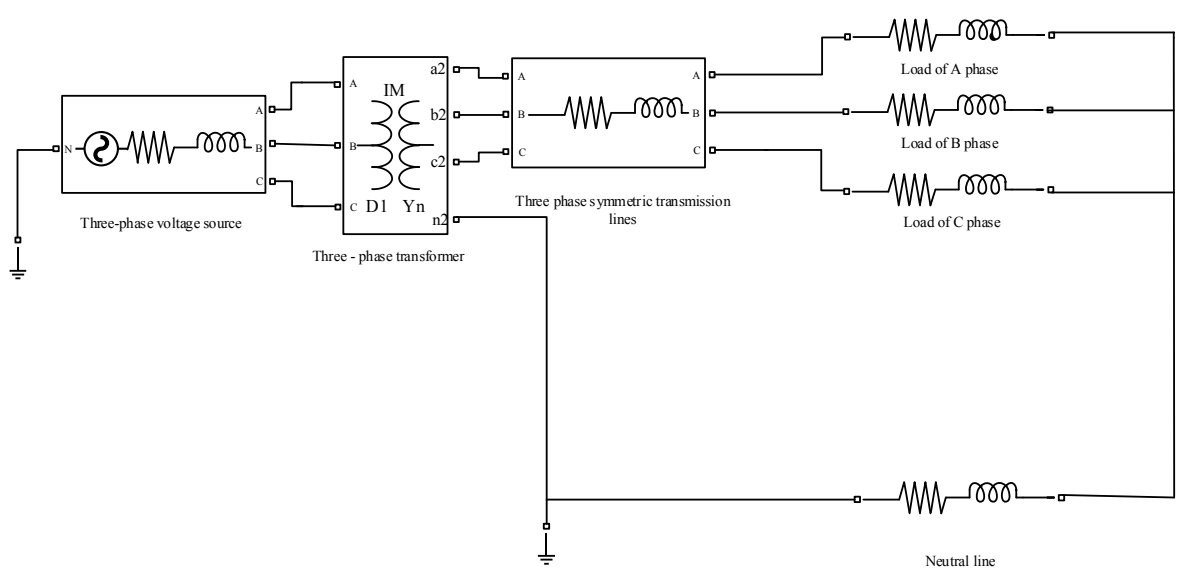

Figure 1. The equivalent resistance model for the low-voltage distribution network.

Table 2 is the results comparing the simulation data with the theoretical results when three-phase load is unbalanced. $\mathrm{P}_{\mathrm{TH}}$ represents the theoretical results of the power loss of the transformer and the line for three cases. $\mathrm{P}_{\mathrm{SIM}}$ represents the simulated results of the power loss of the transformer and the line for three cases. From Table 2 we find that the theoretical results are in agreement with the simulation results. The calculation data is very close to the simulation data and the deviation of the additional loss between calculation and simulation is guaranteed within $6 \%$, which verifies the accuracy of the theoretical derivation.

Table 1. A, B and C three phase load distribution for equilibrium case and different unbalanced cases.

\begin{tabular}{lllll}
\hline \multirow{2}{*}{ Phase line } & $\begin{array}{l}\text { Three-phase } \\
\text { equilibrium (kVA) }\end{array}$ & $\begin{array}{l}\text { Case I } \\
\text { (kVA) }\end{array}$ & $\begin{array}{l}\text { Case II } \\
\text { (kVA) }\end{array}$ & $\begin{array}{l}\text { Case III } \\
\text { (kVA) }\end{array}$ \\
\hline A & 20 & 24 & 22 & 22 \\
B & 20 & 18 & 22 & 20 \\
C & 20 & 18 & 16 & 18 \\
\hline
\end{tabular}

Table 2. Simulation data compared with the theoretical value when three-phase load is unbalanced.

\begin{tabular}{lll}
\hline Load distribution type & $\mathbf{P}_{\mathrm{Th}}(\mathbf{W})$ & $\mathbf{P}_{\text {sim }}(\mathbf{W})$ \\
\hline Case I & 554 & 586 \\
Case II & 620 & 659 \\
Case III & 614 & 637 \\
\hline
\end{tabular}

The distribution of the three-phase load is adjusted under the condition that the total load is unchanged, so that the relationship between the maximum current unbalance and the additional loss of the low-voltage distribution network under the above three different load distribution conditions is obtained.

Table 3 gives the simulated data for the power loss and additional loss of the transformer and the transmission line when the three-phase load is unbalanced. According to the Table 3, in the above three cases, When the three-phase load is unbalanced in low voltage distribution network, under the condition that the total three-phase load is not changed, when changing the three-phase load distribution, it is found that when the phases A and B are heavy and C phase is light (case II), the additional loss is the largest. The situation should be avoided in the actual application. Additional losses are smallest in three cases when phase A heavy load, phase B average load and phase $\mathrm{C}$ light load (case III). The power loss in the neutral line is also calculated and is not listed here. For the neutral current, When the phase A overload, phase $\mathrm{C}$ light load and phase B is average load (case III), neutral loss is the largest, and the difference between the other two is small. In a word the three-phase imbalance has great influence on the additional power loss of the transformer and the transmission line.

Table 3. Simulated addtional loss and l loss of the transformer and the line for three-phase load imbalance.

\begin{tabular}{lllll}
\hline Load distribution type & $\Delta \mathbf{P}_{\mathbf{T}}(\mathbf{W})$ & $\Delta \mathbf{P}_{\mathbf{L}}(\mathbf{W})$ & $\mathbf{P}_{\mathbf{T}}(\mathbf{W})$ & $\mathbf{P}_{\mathbf{L}}(\mathbf{W})$ \\
\hline Case I & 8.4 & 25.3 & 138.4 & 448.5 \\
Case II & 10.1 & 36.3 & 155.2 & 504.3 \\
Case III & 6.6 & 17.7 & 154.6 & 483.7 \\
\hline
\end{tabular}

Figure 2 shows the total additional loss of the simulation data comparing with the theoretical results of a low-voltage distribution network for different loads with the different maximum current unbalance coefficient. The calculation data is very close to the simulation data and the results guarantee the accuracy of the theoretical derivation.

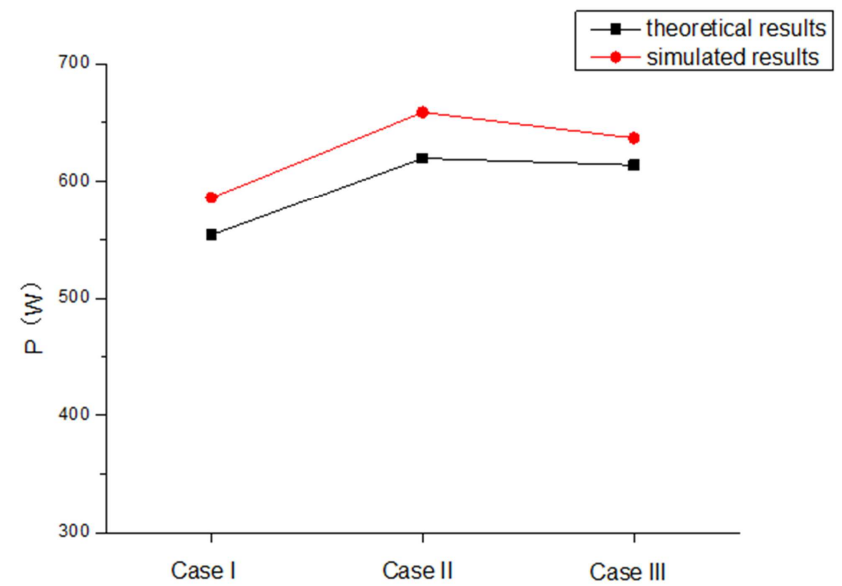

Figure 2. The sumulated results and the theoretical results for different cases. 


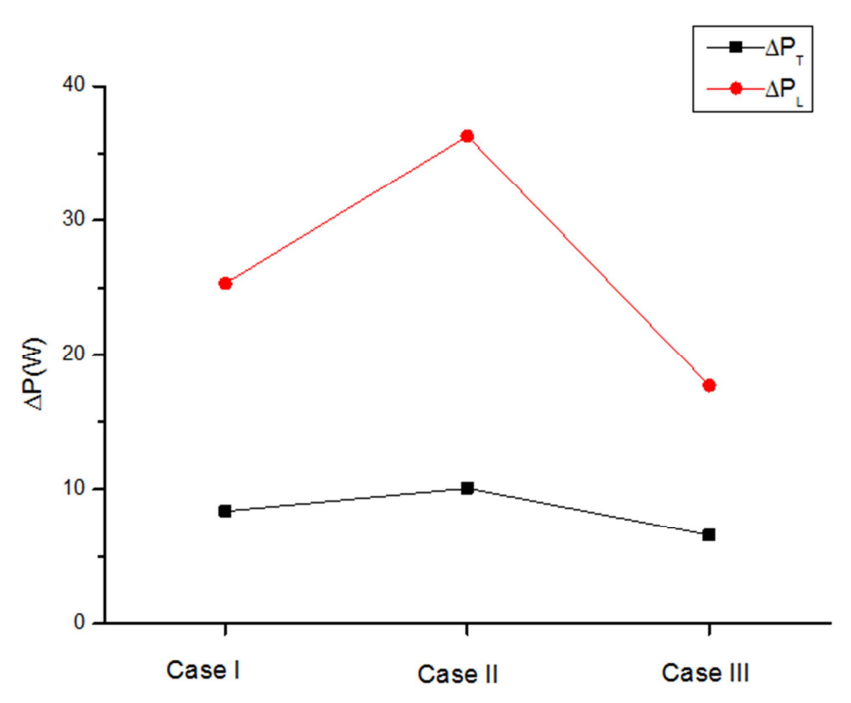

Figure 3. Relationship curve between additional loss for different unbalanced cases.

Figure 3 gives the plots of the additional loss of the transformer and the transmission line for different unbalanced cases. As can be seen from Figure 3, the incremental values of the loss are different for different unbalanced situations. The increment curve is the largest in the second case just as the absolute value of the power loss is also the largest in three cases. For the case I, the total loss is smaller than the total loss of the case II, but the additional loss is greater than the additional loss of the case II. The result shows that the additional loss is not proportional to the total loss both for the transformer and the line.

Table 4. $A, B$ and $C$ three phase unbalanced load distribution with increasing unbalanced degree.

\begin{tabular}{llll}
\hline Phase line & Case III(kVA) & Case IV (kVA) & Case V (kVA) \\
\hline A & 22 & 23 & 24 \\
B & 20 & 20 & 20 \\
C & 18 & 17 & 16 \\
\hline
\end{tabular}

Table 5. Additional loss and increment ratio of the transformer and the line for three cases with different unbalanced degree.

\begin{tabular}{lllll}
\hline Load distribution type & $\Delta \mathbf{P}_{\mathbf{T}}(\mathbf{W})$ & $\Delta \mathbf{P}_{\mathbf{L}}(\mathbf{W})$ & $\delta_{\mathrm{T}}$ & $\delta_{\mathrm{L}}$ \\
\hline Case III & 6.6 & 17.7 & $4.3 \%$ & $3.7 \%$ \\
Case IV & 9.4 & 24.5 & $6.0 \%$ & $5.0 \%$ \\
Case V & 14.5 & 31.1 & $9.2 \%$ & $6.4 \%$ \\
\hline
\end{tabular}

Table 4 gives three cases of unbalanced load with increasing unbalanced degree. Case III is the same as before. The load of phase $A$ in case IV and case $V$ is greater than the value in case III. The load of phase $\mathrm{C}$ in case IV and case $\mathrm{V}$ is smaller than the value in case III which guarantee the total load is the same. Thus the unbalanced degree is greater in case IV and case $\mathrm{V}$ than the case III. Table 5 gives the additional loss and increment ratio of the transformer and the line for the three cases in Table 4. The additional loss and the increment ratio of loss for the transformer and the line in case IV or V are greater than the value in case III. The results show that both the additional loss and the increment ratio of loss for the transformer and the line are increasing with the increase of the unbalanced degree.

The results for the additional loss and the increment ratio of loss for the transformer and the line are plotted in Figure 4. The additional loss of line is greater than the additional loss of the transformer for each case. As the unbalanced degree is increasing, both the additional loss and the increment ratio of loss for the transformer and the line are enhanced.

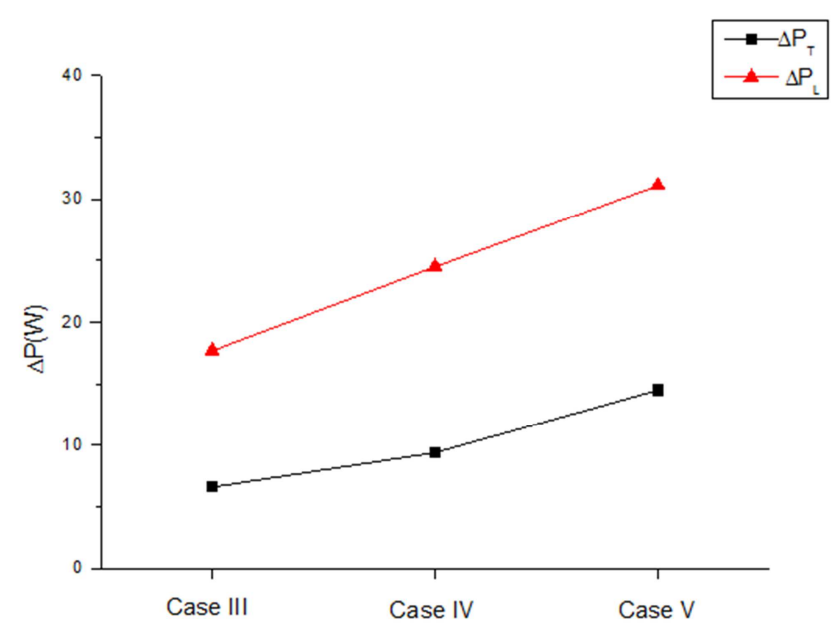

Figure 4. Relationship curve between additional loss with the three unbalanced cases with increasing unbalanced degree.

\section{Conclusion}

Imbalance in power grid is a main aspect of power quality. The power distribution network for single-phase load and uneven distribution in three-phase unbalanced transformer not only cause the insufficient output, overload damage, but also the additional power loss. The degree of current imbalance can influence the additional loss of the equipment such as the transformer and the transmission line in the low voltage distribution network. We verify that the additional loss of the transformer and the transmission line in the low-voltage distribution network for different three phase unbalanced situations. The results show that the three-phase unbalance has great influence on the additional loss. The calculation data is very close to the simulation data and the accuracy of the theoretical derivation is verified. The increase value is the largest one in the second case in which phase $\mathrm{A}$ and $\mathrm{B}$ have heavy load and phase $\mathrm{C}$ is light load just as the absolute value of the power loss in this case is also the largest in three cases. The additional loss is not proportional to the total loss both for the transformer and the line. For case III, one phase is heavy, one phase is light and the last phase is the average one. If the unbalanced degree in case IV and case $\mathrm{V}$ is greater than the value in case III, the results show that both the additional loss and the increment ratio of loss for the transformer and the line are enhanced with the increase of the unbalanced degree.

With the advance of energy saving and consumption reduction, the accurate calculation model of additional loss caused by composite power quality is of great practical significance. Based on the study of this article, we should develop and manage devices and preventive measures as soon 
as possible. From the angle of power loss, the management of power quality can not only be limited to the control of a single index, but also should be comprehensively managed from multiple aspects to achieve optimal results.

\section{Acknowledgements}

This work was supported by 'the Fundamental Research Funds for the Central Universities' (2018MS003).

\section{References}

[1] Asl, Dariush Keihan; Seifi, Ali Reza; Rastegar, Mohammad, Optimal energy flow in integrated energy distribution systems considering unbalanced operation of power distribution systems $[\mathrm{J}]$. International journal of electrical power and energy systems. 2020, 121: 106132.

[2] Zhang Yinghui. The power quality problem of power supply and distribution system must be regulated and caused enough attention [J]. Electrical Applications, 2014, 33 (7): 15-16.

[3] Sun Yan, Lin Shenghong, Liu Mingbo. Analysis of optimal power supply capacity of medium voltage distribution network [J]. Southern Power System Technology, 2014, 8 (1): 80-84.

[4] M. Jawad Ghorbani, Hossein Mokhtari. Impact of Harmonics on Power Quality and Losses in Power Distribution Systems [J] International Journal of Electrical and Computer Engineering, 2015, 7 (1): 166-174.

[5] Congling Li, Peng Wang. Yucheng Li, Research and Application of Unbalanced Three-phase Load Control Scheme for Distribution Network [J]. Editorial Department of Journal of Shangluo University, 2018, 2: 74-78.

[6] Feng Cheng, Xu Changbao. Analysis of influence of three-phase current imbalance and harmonics on power grid loss [J]. Power Quality, 2016, (7): 47-51.

[7] Yanliang Wang, Zhishun Yue, Jinquan Wang, Zhang Qi. Analysis of harmonic additional loss and energy saving for reducing loss [J]. Power electronics, 2009 (2): 15-16.

[8] IEEE. IEEE recommended practice for establishing transformer capability when supplying nonsinusoided load currents. Standard ANSI/IEEE C57. 110-1986.

[9] Zhang An-hong. Research of Loss and Design for Transformer [D]. Changsha: Hunan University, 2005.

[10] Mabel Ebenezer, Rajkumar Mattacaud Ramachandralal, Chandramohanan Nair Padmanabha Pillai Sarasamma. Study and Analysis of the Effect of Harmonics on the Hot Spot Temperature of a Distribution Transformer Using Finite-Volume Method [J]. Electric Power Components and Systems Electric Power Components and Systems, 2015: 2251-2261.

[11] Yang Xiao-ming. Influence Analysis on $10 \mathrm{kV}$ Distribution Transformer's Los s [J]. Hunan Water Conservancy and Electric Power, 2001, (3): 33-34.

[12] Ruan Quan-rong, Wang Liang, Shi Wei. A Kind of Transformer's Nonlinear Model Considering Loss [J]. Transaction of Xi'an Jiaotong University, 2006, 40 (10).

[13] Zhang Jianzhong, Cheng Ming, Zhu Xiaoyong. A novel three-phase doubly salient permanent magnet generator [C]. ICEMS, Nanjing, China, 2005: 407-411.

[14] Pi Jianghong, Zhang Tiqiang, Ming Hafting. Energy consumption calculation and energy saving measures of high-voltage transmission lines [J]. Energy and Energy Conservation, 2013, 10 (97): 60-61.

[15] Wang Yanliang, Yue Zhishun, Wang Jingquan, et al. Analyzing the addition loss of harmonic and how to reducing it for energy saving [J]. Electrical Engineering, 2009, 2 (3): 15-19. 